\title{
Robust Time-Consistent Portfolio Selection for an Investor under CEV Model with Inflation Influence
}

\author{
Peng Yang iD ${ }^{1,2}$ \\ ${ }^{1}$ School of Science, Xijing University, Xi'an 710123, China \\ ${ }^{2}$ School of Mathematics and Statistics, Xi'an Jiaotong University, Xi'an 710049, China \\ Correspondence should be addressed to Peng Yang; yangpeng511@163.com
}

Received 6 May 2020; Accepted 29 May 2020; Published 17 June 2020

Guest Editor: Wenguang Yu

Copyright (c) 2020 Peng Yang. This is an open access article distributed under the Creative Commons Attribution License, which permits unrestricted use, distribution, and reproduction in any medium, provided the original work is properly cited.

A robust time-consistent optimal investment strategy selection problem under inflation influence is investigated in this article. The investor may invest his wealth in a financial market, with the aim of increasing wealth. The financial market includes one riskfree asset, one risky asset, and one inflation-indexed bond. The price process of the risky asset is governed by a constant elasticity of variance $(\mathrm{CEV})$ model. The investor is ambiguity-averse; he doubts about the model setting under the original probability measure. To dispel this concern, he seeks a set of alternative probability measures, which are absolutely continuous to the original probability measure. The objective of the investor is to seek a time-consistent strategy so as to maximize his expected terminal wealth meanwhile minimizing his variance of the terminal wealth in the worst-case scenario. By using the stochastic optimal control technique, we derive closed-form solutions for the optimal time-consistent investment strategy, the probability scenario, and the value function. Finally, the influences of model parameters on the optimal investment strategy and utility loss function are examined through numerical experiments.

\section{Introduction}

Nowadays, portfolio selection is a very important research topic in mathematical finance. As we all know, Markowitz [1] pioneered this research topic. He measured the investment gain and risk by expectation and variance, respectively. Nowadays, scholars call this method as the mean-variance (MV) criterion. $\mathrm{Li}$ and $\mathrm{Ng}$ [2] and Zhou and $\mathrm{Li}$ [3], respectively, pioneered the multiperiod and the continuoustime MV problem, where the explicit solutions were obtained. Dai et al. [4] studied the continuous-time MV problem with transaction costs. Recently, Sun et al. $[5,6]$ studied the MV problem for an insurer.

The aforementioned work under the MV criterion is time inconsistency. That is to say, the optimal strategy made at time $t$ may not be optimal at time $s, s>t$. However, time consistency of strategy is an important and a rational requirement in many practice scenarios. For example, a few years ago, the Chinese government decided to get rid of poverty by 2020 . Naturally, they hope to get rid of poverty at 2020. To the best of our knowledge, Strotz [7] first formally treated time inconsistency. He proposed a game theoretic approach and sought an equilibrium strategy. Thus, the equilibrium strategy is time-consistent. Nowadays, this method becomes a mainstream way to deal with time-inconsistency. Ekeland et al. [8] first proposed the rigorous definition of the equilibrium strategy in a continuous-time framework. Björk and Murgoci [9] studied the time-consistent investment strategy, where the model parameters are controlled by a common Markov process. Li et al. [10] considered the optimal time-consistent MV problem under the Heston model. Yang [11] studied the similar problem, where the aggregate claim and the price are shocked by a common Poisson process. Zeng et al. [12] studied the robust time-consistent strategy under the MV framework. Furthermore, Yang et al. [13] extended the time-consistent MV problem to a new interaction mechanism. For more other detailed and related studies, one can refer to Björk et al. [14], Czichowsky [15], and Kronborg and Steffensen [16]. 
However, most of the aforementioned papers ignore the inflation risk and assume that the risky asset follows a geometric Brownian motion, which is usually contrary to practice investment activities according to many empirical studies. Brennan and Xia [17] studied the asset distribution problem under inflation influence. Han and Huang [18] considered the defined-contribution (DC) pension problem under inflation influence. Kwak and Lim [19] studied the optimal consumption-investment problem under inflation influence. Li et al. [20] studied the time-consistent investment strategy selection problem for a DC pension under partial information and inflation influence.

On the contrary, the CEV model has become popular among academia and practitioners since proposed by Cox and Ross [21]. Gao [22] investigated the DC pension investment problem under the CEV model. Gu et al. [23], Lin and Li [24], and Liang et al. [25] investigated the optimal reinsurance and investment strategy selection problem under the CEV model. Lin and Qian [26] studied the similar problem, where the strategy is time-consistent. For more other detailed and related studies about the CEV model, readers can refer to Li et al. [27] and Zheng et al. [28].

Bearing in mind the aforementioned state of the art, in this article, we will investigate the time-consistent investment strategy under the CEV model with inflation influence. The financial market includes one risk-free asset, one risky asset, and one inflation-indexed bond. The price process of the risky asset is governed by the CEV model. We assume that the investor is ambiguity-averse and worries about uncertainty in model setting. To dispel this concern, he seeks a set of alternative probability measures, which are absolutely continuous to the original probability measure. Then, the new model setting is obtained under the new probability measure. The objective of the investor is to find a time-consistent strategy so as to maximize his expected terminal wealth meanwhile minimizing his variance of the terminal wealth in the worst-case scenario. The problem's solving steps are as follows: first, we provide an extended HJB equation and a verification theorem. Second, by using the stochastic optimal control technique, we derive analytically the optimal timeconsistent investment strategy, the probability scenario, and the value function. Finally, the influences of model parameters on the optimal investment strategy and utility loss function are examined through numerical experiments.

Compared with some related current research studies, our main contributions are given as follows:

(i) We consider a general financial market, which includes one risk-free asset, one risky asset, and one inflation-indexed bond. The price process of the risky asset is governed by the CEV model.

(ii) We study the time-consistent investment problem under inflation influence.

(iii) Closed-form solutions for the optimal time-consistent robust investment strategy, optimal probability scenario, and value function are derived.

(iv) The influences of model parameters on the optimal time-consistent investment strategy and utility loss function are systematically examined through numerical experiments.

The remainder of this article is arranged as follows. In Section 2, we describe the model setup. In Section 3, an optimal robust time-consistent investment strategy selection problem is formulated. In Section 4, the closed-form solutions for the time-consistent optimal investment strategy and optimal probability scenario are derived. In Section 5, the influences of model parameters on the optimal timeconsistent investment strategy and utility loss function are examined through numerical experiments. The final section summarizes this article.

\section{Model Setup}

We construct a financial model and present some basic assumptions in this section. All stochastic processes and random variables, mentioned later, are defined on a filtered complete probability space $\left(\Omega, \mathscr{F}, \mathscr{F}_{t}, P\right)$. We assume that $\mathscr{F}:=\left\{\mathscr{F}_{t}, t \geq 0\right\}$ is right-continuous and is complete with respect to $P$. $\mathscr{F}_{t}$ stands for the information acquired by the investor up to time $t$. We assume that there are no market frictions in trading.

2.1. Financial Market. A financial market with the inflation risk is given in this section. To the best of our knowledge, consumer price index (CPI) is often used to describe the inflation rate in economics and academic research. CPI can be seen as a price level process. In this article, the inflation price level $P_{0}(t)$ satisfies the following process:

$$
\mathrm{d} P_{0}(t)=P_{0}(t)\left[\mu_{1} \mathrm{~d} t+\sigma_{1} \mathrm{~d} W_{1}(t)\right]
$$

where $\mu_{1}>0$ represents the instantaneous expected rate of the inflation, $\sigma_{1}>0$ stands for the volatility of the inflation, and $W_{1}(t)$ stands for a standard Brownian motion.

We assume that there are three assets available for the investor: one risk-free asset, one risky asset, and one inflation-indexed bond. The price process $P_{1}(t)$ of the risk-free asset is given by

$$
\mathrm{d} P_{1}(t)=r_{1} P_{1}(t) \mathrm{d} t
$$

Here, $r_{1}>0$ represents the interest rate of the risk-free asset. The price process $S(t)$ of the risky asset satisfies the following CEV model:

$$
\mathrm{d} S(t)=S(t)\left[\mu_{2} \mathrm{~d} t+\sigma_{2} S^{\beta}(t) \mathrm{d} W_{2}(t)\right], S(0)=s_{0} .
$$

Here, $\mu_{2} \geq r_{1}$ stands for the appreciation rate, $\sigma_{2}>0$ represents the price volatility, $W_{2}(t)$ represents a standard Brownian motion, and $\beta$ is the constant elasticity parameter. We assume $W_{1}(t)$ and $W_{2}(t)$ are mutually independent.

Remark 1. The parameter $\beta$ can take all real numbers. Obviously, if $\beta=0$, the CEV model will degenerate to a geometric Brownian motion. When the CEV model is proposed, $\beta$ is assumed to be negative. Emanuel and Macbeth [29] proved that $\beta$ can also be assumed to be 
positive. The CEV model has no jump, i.e., we do not consider the jump risk in this article. We can also consider the price process with the jump. Then, the exponential Lévy model is a good choice. For the exponential Lévy model, readers can refer to $\mathrm{Yu}$ et al. [30] and Zhang et al. [31].

The third asset to invest in is the inflation-indexed bond. Similar to Kwak and Lim [19], the price $P(t)$ of the inflationindexed bond is given by

$$
\frac{\mathrm{d} P(t)}{P(t)}=\left[r_{2} \mathrm{~d} t+\frac{\mathrm{d} P_{0}(t)}{P_{0}(t)}\right]=\left(r_{2}+\mu_{1}\right) \mathrm{d} t+\sigma_{1} \mathrm{~d} W_{1}(t) .
$$

Here, $r_{2}>0$ represents the real interest rate, and $r_{2}+$ $\mu_{1}>r_{1}$ stands for the appreciation rate.

2.2. Inflation-Adjusted Wealth Process. Let $u_{1}(t)$ and $u_{2}(t)$, respectively, denote the proportions of the wealth invested in the risky asset and the inflation-indexed bond at time $t$, and the remainder of the proportion is invested in the risk-free asset. At time $t$, the investor (here, we only consider an investor. To reflect the general situation, one can consider $n$ investors. For example, Yang et al. [13], Espinosa and Touzi [32], and Yu et al. [33] studied the optimal control problem for $n$ agents) can choose the proportions $u_{1}(t)$ and $u_{2}(t)$ as control strategies; we denote them as $u(t)=\left(u_{1}(t), u_{2}(t)\right)$. For each strategy $u(t)$, the wealth process $R_{t}^{u}$ can be described as

$$
\begin{aligned}
\frac{\mathrm{d} R_{t}^{u}}{R_{t}^{u}}= & {\left[1-u_{1}(t)-u_{2}(t)\right] \frac{\mathrm{d} P_{1}(t)}{P_{1}(t)}+u_{1}(t) \frac{\mathrm{d} S(t)}{S(t)}+u_{2}(t) \frac{\mathrm{d} P(t)}{P(t)} } \\
= & {\left[r_{1}+u_{1}(t)\left(\mu_{2}-r_{1}\right)+u_{2}(t)\left(r_{2}+\mu_{1}-r_{1}\right)\right] \mathrm{d} t } \\
& +u_{1}(t) \sigma_{2} S^{\beta}(t) \mathrm{d} W_{2}(t)+u_{2}(t) \sigma_{1} \mathrm{~d} W_{1}(t) .
\end{aligned}
$$

Denote

$$
X_{t}^{u}=\frac{R_{t}^{u}}{P_{0}(t)}
$$

By applying Itô's formula, $X_{t}^{u}$ can be described as

$$
\begin{aligned}
\mathrm{d} X_{t}^{u}= & \frac{1}{P_{0}(t)} \mathrm{d} R_{t}^{u}+R_{t}^{u} \mathrm{~d} \frac{1}{P_{0}(t)}+d\left\langle R_{t}^{u}, \frac{1}{P_{0}(t)}\right\rangle \\
= & X_{t}^{u}\left\{\left[r_{1}+\sigma_{1}^{2}-\mu_{1}+u_{1}(t)\left(\mu_{2}-r_{1}\right)\right.\right. \\
& \left.+u_{2}(t)\left(r_{2}+\mu_{1}-r_{1}-\sigma_{1}^{2}\right)\right] \mathrm{d} t \\
& \left.+u_{1}(t) \sigma_{2} S^{\beta}(t) \mathrm{d} W_{2}(t)+\left(u_{2}(t)-1\right) \sigma_{1}(t) \mathrm{d} W_{1}(t)\right\} .
\end{aligned}
$$

Here, $X_{t}^{u}$ is the real wealth with stripping out inflation.

To facilitate solving the optimization problem in Section 3 , we define the following notations:

$$
\begin{cases}\pi_{1}(t)=u_{1}(t) X_{t}^{u}, \pi_{2}(t)=\left[u_{2}(t)-1\right] X_{t}^{u}, & \pi(t)=\left(\pi_{1}(t), \pi_{2}(t)\right), \\ \widetilde{r}_{1}=r_{1}+\sigma_{1}^{2}-\mu_{1}, \widetilde{r}_{2}=r_{2}+\mu_{1}-r_{1}-\sigma_{1}^{2}, & \tilde{r}=\widetilde{r}_{1}+\widetilde{r}_{2} .\end{cases}
$$

Then, the real wealth $X_{t}^{\pi}$ can be described as

$$
\begin{aligned}
\mathrm{d} X_{t}^{\pi}= & {\left[\widetilde{r} X_{t}^{\pi}+\pi_{1}(t)\left(\mu_{2}-r_{1}\right)+\pi_{2}(t) \widetilde{r}_{2}\right] \mathrm{d} t } \\
& +\pi_{1}(t) \sigma_{2} S^{\beta}(t) \mathrm{d} W_{2}(t)+\pi_{2}(t) \sigma_{1} \mathrm{~d} W_{1}(t) .
\end{aligned}
$$

\section{Problem Formulation}

MV investment strategy selection problem as a classic optimization problem can be given as follows:

$$
\sup _{\pi}\left\{E_{0, x_{0}, s_{0}}\left[X_{T}^{\pi}\right],-\operatorname{Var}_{0, x_{0}, s_{0}}\left[X_{T}^{\pi}\right]\right\} \text {. }
$$

Here, $\quad E_{0, x_{0}, s_{0}}[\cdot]=E\left[X_{0}^{\pi}=x_{0}, S(0)=s_{0}\right], \quad \operatorname{Var}_{0, x_{0}, s_{0}}[\cdot]=$ $\operatorname{Var}\left[X_{0}^{\pi}=x_{0}, S(0)=s_{0}\right], x_{0}$ is the investor's initial wealth, and $T$ is the termination time of the investment. Obviously, the objective of the investor is to find an investment strategy so as to maximize his expected terminal wealth meanwhile minimizing his variance of the terminal wealth. It is well known that this problem is equivalent to the following problem:

$$
\sup _{\pi}\left\{E_{0, x_{0}, s_{0}}\left[X_{T}^{\pi}\right]-\frac{\gamma}{2} \operatorname{Var}_{0, x_{0}, s_{0}}\left[X_{T}^{\pi}\right]\right\},
$$

where $\gamma>0$ represents the risk-aversion parameter. According to Björk and Murgoci [9] and Kronborg and
Steffensen [16], we know that the optimal strategy of (11) is a precommitment strategy, which is time-inconsistent.

As we explained in the introduction, one usually takes into account the time-consistent strategy. Similar to Yang [11], Lin and Qian [26], and other related papers, we consider the following problem:

$$
\sup _{\pi}\left\{E_{t, x, s}\left[X_{T}^{\pi}\right]-\frac{\gamma}{2} \operatorname{Var}_{t, x, s}\left[X_{T}^{\pi}\right]\right\}
$$

where $\quad E_{t, x, s}[\cdot]=E\left[X_{t}^{\pi}=x, S(t)=s\right]$ and $\quad \operatorname{Var}_{t, x, s}[\cdot]=$ $\operatorname{Var}\left[X_{t}^{\pi}=x, S(t)=s\right]$. The target is to develop the corresponding equilibrium investment strategy, which is timeconsistent.

Problem (12) is the traditional MV problem, where the investor is ambiguity-neutral. That is, he fully believes in the model defined under the probability measure $P$. In the actual economic activities, the investor often is ambiguity-averse and wants to protect himself against worst-case scenarios. Similar to Zeng et al. [12], Maenhout [34, 35], Chen and Yang [36], and other papers, we incorporate ambiguity aversion into MV problem (12). Since the investor is ambiguity-averse, he may doubt the model defined under the probability measure $P$. Hence, we defined an alternative probability measure $Q$, which is absolutely continuous to $P$. All such $Q$ are denoted by $Q$, that is, 


$$
Q:=\{Q \mid Q \sim P\}
$$

Now, we define an admissible strategy.

Definition 1. For any fixed $t \in[0, T]$, an investment strategy $\pi(t)=\left(\pi_{1}(t), \pi_{2}(t)\right)$ is called an admissible strategy for the ambiguity-averse investor (AAI) if it satisfies the following:

(i) $\pi_{1}(t)$ and $\pi_{2}(t)$ are progressively measurable with respect to $\mathscr{F}_{t}$

(ii) $E_{t, x, s}^{\mathrm{Q}^{*}}\left[\int_{0}^{T} \pi_{1}^{2}(t) S^{2 \beta} \mathrm{d} t\right]<\infty$ and $E_{t, x, s}^{\mathrm{Q}^{*}}\left[\int_{0}^{T} \pi_{2}^{2}(t) \mathrm{d} t\right]<\infty$, where $Q^{*}$ is the probability measure under the worst-case scenario and $E_{t, x, s}^{Q^{*}}[\cdot]=E_{t, x, s}^{Q^{*}}\left[\cdot \mid X_{t}^{\pi}=x\right.$, $S(t)=s]$

(iii) Equation (9) with respect to $\pi(t)$ has a unique strong solution

We denote all such admissible investment strategies on the time interval $[0, T]$ as $\Pi$.

To transform model (9) from the probability measure $P$ to the probability measure $Q$, we define a process $\left\{\theta(t)=\left(\theta_{1}(t), \theta_{2}(t)\right) \mid t \in[0, T]\right\}$, which satisfies the following:

(i) $\theta_{1}(t)$ and $\theta_{2}(t)$ are progressively measurable with respect to $\mathscr{F}_{t}$

(ii) $E\left[\exp \left\{(1 / 2) \int_{0}^{T}\left[\theta_{1}^{2}(t)+\theta_{2}^{2}(t)\right] \mathrm{d} t\right\}\right]<\infty$

All such $\theta(t)$ on the time interval $[0, T]$ are denoted by $\Theta$. For any $\theta \in \Theta$, we define a real-valued process $\left\{Z^{\theta}(t) \mid t \in[0, T]\right\}$ on $\left(\Omega, \mathscr{F}_{t}, \mathscr{F}_{t}, P\right)$ as

$$
\begin{aligned}
Z^{\theta}(t):= & \exp \left\{\int_{0}^{t} \theta_{1}(s) \mathrm{d} W_{1}(s)+\int_{0}^{t} \theta_{2}(s) \mathrm{d} W_{2}(s)\right. \\
& \left.-\frac{1}{2} \int_{0}^{t} \theta_{1}^{2}(s) \mathrm{d} s-\frac{1}{2} \int_{0}^{t} \theta_{2}^{2}(s) \mathrm{d} s\right\} .
\end{aligned}
$$

Then, $Z^{\theta}(t)$ is a martingale with respect to $P$. In the following, we define a new probability measure $Q$, which is given by

$$
\left.\frac{\mathrm{dQ}}{\mathrm{d} P}\right|_{\mathscr{F}_{T}}:=Z^{\theta}(T)
$$

From the definition of $Q$, it is clear that $Q$ is absolutely continuous to $P$.

For any $\theta \in \Theta$, we, respectively, define two new processes $W_{1}^{Q}(t)$ and $W_{2}^{Q}(t)$ by

$$
\begin{aligned}
& \mathrm{d} W_{1}^{\mathrm{Q}}(t)=\mathrm{d} W_{1}(t)-\theta_{1}(t) \mathrm{d} t \\
& \mathrm{~d} W_{2}^{\mathrm{Q}}(t)=\mathrm{d} W_{2}(t)-\theta_{2}(t) \mathrm{d} t .
\end{aligned}
$$

According to Girsanov's theorem, $W_{1}^{Q}(t)$ and $W_{2}^{Q}(t)$ are standard Brownian motions with respect to Q. Furthermore, the wealth process (9) under $Q$ can be rewritten as

$$
\begin{aligned}
\mathrm{d} X_{t}^{\pi}= & {\left[\widetilde{r} X_{t}^{\pi}+\pi_{1}(t)\left(\mu_{2}-r_{1}\right)+\pi_{2}(t) \widetilde{r}_{2}\right.} \\
& \left.+\pi_{1}(t) \theta_{2}(t) \sigma_{2} S^{\beta}+\pi_{2}(t) \theta_{1}(t) \sigma_{1}\right] \mathrm{d} t \\
& +\pi_{1}(t) \sigma_{2} S^{\beta} \mathrm{d} W_{2}^{Q}(t)+\pi_{2}(t) \sigma_{1} \mathrm{~d} W_{1}^{Q}(t),
\end{aligned}
$$

and corresponding CEV model (3) becomes

$$
\mathrm{d} S(t)=S(t)\left[\left(\mu_{2}+\theta_{2}(t) \sigma_{2} S^{\beta}(t)\right) \mathrm{d} t+\sigma_{2} S^{\beta}(t) \mathrm{d} W_{2}^{Q}(t)\right] .
$$

In the following, we modify MV problem (12) under the worst-case scenario. Through modifying MV problem (12), we shall deal with a robust time-consistent MV strategy selection problem as follows:

$$
\sup _{\pi \in \Pi} V^{\pi}(t, x, s)=\sup _{\pi \in \Pi}\left\{\inf _{Q \in \mathbb{Q}} \widehat{V}^{\pi, Q}(t, x, s)\right\} .
$$

Here,

$$
\begin{aligned}
& \widehat{V}^{\pi, Q}(t, x, s)= E_{t, x, s}^{Q}\left\{\int_{t}^{T}\left[\frac{\left(\theta_{1}(v)\right)^{2}}{2 \phi_{1}(v)}+\frac{\left(\theta_{2}(v)\right)^{2}}{2 \phi_{2}(v)}\right] \mathrm{d} v\right\} \\
&+E_{t, x, s}^{Q}\left[X_{T}^{\pi}\right]-\frac{\gamma}{2} \operatorname{Var}_{t, x, s}^{Q}\left[X_{T}^{\pi}\right], \\
& V^{\pi}(t, x, s)=\inf _{Q \in \mathbb{Q}} \widehat{V}^{\pi, Q}(t, x, s),
\end{aligned}
$$

where $\phi_{1}(t)$ and $\phi_{2}(t)$ are nonnegative and stand for the investor's ambiguity aversion with respect to the model under $P$. The larger $\phi_{1}(t)$ and $\phi_{2}(t)$ are, the more the model under $Q$ will deviate from the model under $P$. Therefore, AAI's ambiguity aversion is an increasing function of $\phi_{1}(t)$ and $\phi_{2}(t)$. To embody some good properties of the model under $P$, AAI deviation from $P$ is penalized by the first two terms in (20). The penalty terms depend on the relative entropy. The increase in relative entropy from $t$ to $t+d t$ equals

$$
\frac{1}{2}\left[\left(\theta_{1}(t)\right)^{2}+\left(\theta_{2}(t)\right)^{2}\right] \mathrm{d} t
$$

The proof of (22) is similar to that in Appendix A in Zeng et al. [12]; we omit it here.

To obtain the time-consistent investment strategy, we present the definition of the equilibrium strategy.

Definition 2. For $\forall(t, x, s) \in[0, T] \times R \times R^{+}$, we choose an admissible strategy $\pi^{*}(t, x, s) \in \Pi$. Then, through choosing three real numbers $\widetilde{a} \in R^{+}, \widetilde{b} \in R$, and $\varsigma>0$, we define a new strategy by

$$
\pi^{\varsigma}(l, \tilde{x}, \widetilde{s})= \begin{cases}(\tilde{a}, \tilde{b}) & \text { for }(l, \tilde{x}, s) \in[t, t+\varsigma) \times R \times R^{+} \\ \pi^{*}(l, \tilde{x}, s), & \text { for }(l, \tilde{x}, s) \in[t+\varsigma, T] \times R \times R^{+}\end{cases}
$$

If for any $(\widetilde{a}, \widetilde{b}) \in R^{+} \times R$ and $(t, x, s) \in[0, T] \times R \times R^{+}$, we have

$$
\liminf _{\varsigma \rightarrow 0} \frac{V^{\pi^{*}}(t, x, s)-V^{\pi^{\varsigma}}(t, x, s)}{\varsigma} \geq 0,
$$

which holds; then, $\pi^{*}(t, x, s)$ is called an equilibrium strategy, and the equilibrium value function $V(t, x, s)$ is given by

$$
V(t, x, s)=V^{\pi^{*}}(t, x, s) .
$$


As evidenced by many references such as Björk and Murgoci [9] and Yang et al. [13], the equilibrium strategy defined by Definition 2 is time-consistent. Therefore, in the following, we call the equilibrium strategy and the equilibrium value function as the optimal time-consistent strategy and the optimal value function. Thus, the AAI's aim is to find an optimal time-consistent investment strategy to solve robust optimization problem (19).

For convenience, we define two notations:

$$
\begin{aligned}
C^{1,2,2}\left([0, T] \times R \times R^{+}\right)= & \left\{\varphi(t, x, s) \mid \text { for } \forall[0, T] \times R \times R^{+},\right. \\
& \varphi_{x}(t, x, s), \varphi_{x x}(t, x, s), \varphi_{s}(t, x, s), \\
& \left.\varphi_{s s}(t, x, s) \text { are continuous }\right\},
\end{aligned}
$$

and the usual infinitesimal generator $\mathscr{A}^{\pi, \theta}$ for the wealth process (17) is given by

$$
\begin{aligned}
\mathscr{A}^{\pi, \theta}(\varphi(t, x, s))= & \varphi_{t}(t, x, s)+\left[\widetilde{r} x+\pi_{1}(t)\left(\mu_{2}-r_{1}\right)+\pi_{2}(t) \widetilde{r}_{2}+\pi_{2}(t) \theta_{1}(t) \sigma_{1}+\pi_{1}(t) \theta_{2}(t) \sigma_{2} s^{\beta}\right] \varphi_{x}(t, x, s) \\
& +\frac{1}{2}\left[\pi_{1}^{2}(t) \sigma_{2}^{2} s^{2 \beta}+\pi_{2}^{2}(t) \sigma_{1}^{2}\right] \varphi_{x x}(t, x, s)+\left[\mu_{2} s+\theta_{2}(t) \sigma_{2} s^{\beta+1}\right] \varphi_{s}(t, x, s)+\frac{1}{2} \sigma_{2}^{2} s^{2 \beta+2} \varphi_{s s}+\pi_{1}(t) \sigma_{2}^{2}(t) s^{2 \beta+1} \varphi_{s x}(t, x, s),
\end{aligned}
$$

where $\varphi(t, x, s) \in C^{1,2,2}\left([0, T] \times R \times R^{+}\right), \pi \in \Pi$, and $\theta \in \Theta$.

To ensure the strategy obtained from robust $\mathrm{MV}$ problem (19) is optimal, we present the following verification theorem. The proof of this theorem is similar to that in Theorem 7.1 of Björk and Murgoci [9], Theorem 4.1 of Yang et al. [13], and other papers, so we omit it here.
Theorem 1. For robust $M V$ problem (19), if there exist two functions $W(t, x, s)$ and $g(t, x, s)$ satisfying the following extended $H J B$ equation

$$
\begin{gathered}
\sup _{\pi \in \Pi} \inf _{Q \in \mathcal{Q}}\left\{\mathscr{A}^{\pi, \theta}(W(t, x, s))-\mathscr{A}^{\pi, \theta}\left(\frac{\gamma}{2} g^{2}(t, x, s)\right)+\gamma g(t, x, s) \mathscr{A}^{\pi, \theta}(g(t, x, s))+\frac{\left(\theta_{1}(t)\right)^{2}}{2 \phi_{1}(t)}+\frac{\left(\theta_{2}(t)\right)^{2}}{2 \phi_{2}(t)}\right\}=0, W(T, x, s)=x, \\
\mathscr{A}^{\pi^{*}, \theta^{*}}(g(t, x, s))=0, g(T, x, s)=x \\
\left(\pi^{*}, \theta^{*}\right)=\operatorname{argsup}_{\pi \in \Pi} \inf _{Q \in \mathscr{Q}}\left\{\mathscr{A}^{\pi, \theta}(W(t, x, s))-\mathscr{A}^{\pi, \theta}\left(\frac{\gamma}{2} g^{2}(t, x, s)\right)+\gamma g(t, x, s) \mathscr{A}^{\pi, \theta}(g(t, x, s))+\frac{\left(\theta_{1}(t)\right)^{2}}{2 \phi_{1}(t)}+\frac{\left(\theta_{2}(t)\right)^{2}}{2 \phi_{2}(t)}\right\}
\end{gathered}
$$

then $V(t, x, s)=W(t, x, s), E_{t, x, s}^{Q}\left[X_{T}^{\pi^{*}}\right]=g(t, x, s), \pi^{*}$ is the optimal robust time-consistent investment strategy for AAI, and $\theta^{*}$ is the optimal probability scenario for the market.

\section{The Solution to the Robust MV Problem}

This section is devoted to solve robust MV problem (19). By solving HJB equations (28) and (29), we can derive the solution to robust MV problem (19). To solve robust MV problem (19), similar to Maenhout $[34,35]$ and Chen and Yang [36], this article assumes that $\phi_{1}(t)=\alpha_{1}$ and $\phi_{2}(t)=\alpha_{2}$, where $\alpha_{1}$ and $\alpha_{1}$ are nonnegative.

Now, we give an explicit expression for HJB equation (28).

According to (27), we obtain

$$
\begin{aligned}
\mathscr{A}^{\pi, \theta}\left(\frac{\gamma}{2} g^{2}(t, x, s)\right)= & \gamma g g_{t}+\left[\widetilde{r} x+\pi_{1}(t)\left(\mu_{2}-r_{1}\right)+\pi_{2}(t) \widetilde{r}_{2}+\pi_{2}(t) \theta_{1}(t) \sigma_{1}+\pi_{1}(t) \theta_{2}(t) \sigma_{2} s^{\beta}\right] \gamma g g_{x} \\
& +\frac{1}{2}\left[\pi_{1}^{2}(t) \sigma_{2}^{2} s^{2 \beta}+\pi_{2}^{2}(t) \sigma_{1}^{2}\right]\left(\gamma g_{x}^{2}+\gamma g g_{x x}\right)+\mu_{2} s \gamma g g_{s}+\frac{1}{2} \sigma_{2}^{2} s^{2 \beta+2}\left[\gamma g_{s}^{2}+\gamma g g_{s s}\right] \\
& +\pi_{1}(t) \sigma_{2}^{2} s^{2 \beta+1}\left[\gamma g_{s} g_{x}+\gamma g g_{s x}\right], \\
\gamma g(t, x, s) \mathscr{A}^{\pi, \theta}(g(t, x, s))= & \gamma g g_{t}+\left[\widetilde{r} x+\pi_{1}(t)\left(\mu_{2}-r_{1}\right)+\pi_{2}(t) \widetilde{r}_{2}+\pi_{2}(t) \theta_{1}(t) \sigma_{1}+\pi_{1}(t) \theta_{2}(t) \sigma_{2} s^{\beta}\right] \gamma g g_{x} \\
& +\frac{1}{2}\left[\pi_{1}^{2}(t) \sigma_{2}^{2} s^{2 \beta}+\pi_{2}^{2}(t) \sigma_{1}^{2}\right] \gamma g g_{x x}+\mu_{2} s \gamma g g_{s}+\frac{1}{2} \sigma_{2}^{2} s^{2 \beta+2} \gamma g g_{s s}+\pi_{1}(t) \sigma_{2}^{2}(t) s^{2 \beta+1} \gamma g g_{s x},
\end{aligned}
$$


where $g(t, x, s)$ is abbreviated as $g$ and $g_{t}, g_{x}, g_{x x}, g_{s}, g_{s s}$, and $g_{s x}$ are the partial derivatives of $g$ with respect to the corresponding variables.
Then, HJB equation (28) can be more explicitly expressed as

$$
\begin{aligned}
& \sup _{\pi \in \Pi} \inf _{Q \in Q}\left\{W_{t}+\left[\widetilde{r} x+\pi_{1}(t)\left(\mu_{2}-r_{1}\right)+\pi_{2}(t) \widetilde{r}_{2}+\pi_{2}(t) \theta_{1}(t) \sigma+\pi_{1}(t) \theta_{2}(t) \sigma_{2} s^{\beta}\right] W_{x}\right. \\
& \quad+\frac{1}{2}\left[\pi_{1}^{2}(t) \sigma_{2}^{2} s^{2 \beta}+\pi_{2}^{2}(t) \sigma_{1}^{2}\right]\left(W_{x x}-\gamma g_{x}^{2}\right)+\left[\mu_{2} s+\theta_{2}(t) \sigma_{2} s^{\beta+1}\right] W_{s} \\
& \left.\quad+\frac{1}{2} \sigma_{2}^{2} s^{2 \beta+2}\left(W_{s s}-\gamma g_{s}^{2}\right)+\pi_{1}(t) \sigma_{2}^{2} s^{2 \beta+1}\left(W_{s x}-\gamma g_{s} g_{x}\right)+\frac{\theta_{1}^{2}(t)}{2 \alpha_{1}}+\frac{\theta_{2}^{2}(t)}{2 \alpha_{2}}\right\}=0,
\end{aligned}
$$

where $W(t, x, s)$ is abbreviated as $W$ and $W_{t}, W_{x}, W_{x x}, W_{s}$, $W_{s s}$, and $W_{s x}$ are the partial derivatives of $W$ with respect to the corresponding variables.

Theorem 2. For robust MV problem (19), the optimal robust time-consistent investment strategies are given by

$$
\begin{aligned}
& \pi_{1}^{*}(t)=\frac{\left(\mu_{2}-r_{1}\right)+2 \gamma \beta \sigma_{2}^{2} \bar{B}(t)+2 \alpha_{2} \beta \sigma_{2}^{2} B(t)}{\left(\alpha_{2}+\gamma\right) \sigma_{2}^{2} s^{2 \beta}} e^{-\widetilde{r}(T-t)}, \\
& \pi_{2}^{*}(t)=\frac{\widetilde{r}_{2}}{\left(\alpha_{1}+\gamma\right) \sigma_{1}^{2}} e^{-\tilde{r}(T-t)}
\end{aligned}
$$

The optimal probability scenarios for the market are given by

$$
\begin{aligned}
& \theta_{1}^{*}(t)=-\frac{\alpha_{1} \widetilde{r}_{2}}{\left(\alpha_{1}+\gamma\right) \sigma_{1}}, \\
& \theta_{2}^{*}(t)=\frac{2 \beta \alpha_{2} \sigma_{2} B(t)}{s^{\beta}}-\frac{\alpha_{2}\left[\left(\mu_{2}-r_{1}\right)+2 \gamma \beta \sigma_{2}^{2} \bar{B}(t)+2 \alpha_{2} \beta \sigma_{2}^{2} B(t)\right]}{\left(\alpha_{2}+\gamma\right) \sigma_{2} s^{\beta}}
\end{aligned}
$$

The optimal value function is given by

$$
W(t, x, s)=x e^{\tilde{r}(T-t)}+B(t) s^{-2 \beta}+C(t),
$$

where $C(t)$ is given by $(60)$, and $\bar{B}(t)$ and $B(t)$ are determined by the following ordinary differential equations (ODEs):

$$
\left\{\begin{array}{l}
B^{\prime}(t)-2 \mu_{2} \beta B(t)-2 \sigma_{2}^{2} \gamma \beta^{2} \bar{B}^{2}-2 \alpha_{2} \beta^{2} \sigma_{2}^{2} B^{2}(t)+\frac{\left[\mu_{2}-r_{1}+2 \beta \sigma_{2}^{2}\left(\gamma \bar{B}(t)+\alpha_{2} B(t)\right)\right]^{2}}{2\left(\alpha_{2}+\gamma\right) \sigma_{2}^{2}}=0 \\
\bar{B}^{\prime}(t)-2 \bar{B}(t)\left[\mu_{2} \beta+4 \alpha_{2} \beta^{2} \sigma_{2}^{2} B(t)\right]+\frac{1}{\left(\alpha_{2}+\gamma\right) \sigma_{2}^{2}}\left\{\left[\mu_{2}-r_{1}+2 \sigma_{2}^{2} \alpha_{2} \beta(\bar{B}(t)+B(t))\right] \times\left[\mu_{2}-r_{1}+2 \beta \sigma_{2}^{2}\left(\gamma \bar{B}(t)+\alpha_{2} B(t)\right)\right]\right\} \\
\quad-\frac{\alpha_{2}\left[\mu_{2}-r_{1}+2 \beta \sigma_{2}^{2}\left(\gamma \bar{B}(t)+\alpha_{2} B(t)\right)\right]^{2}}{\left(\alpha_{2}+\gamma\right)^{2} \sigma_{2}^{2}}=0
\end{array}\right.
$$

with boundary conditions $B(T)=0$ and $\bar{B}(T)=0$.

Proof. According to the structure of the wealth dynamics (17) and the boundary conditions $W(T, x, s)=$ $g(T, x, s)=x$, we will seek the solution to HJB equations (28) and (29) with the following parametric form:

$$
W(t, x, s)=A(t) x+B(t) s^{-2 \beta}+C(t),
$$

with boundary conditions

$$
\begin{gathered}
A(T)=1, B(T)=0, C(T)=0, \\
g(t, x, s)=\bar{A}(t) x+\bar{B}(t) s^{-2 \beta}+\bar{C}(t),
\end{gathered}
$$

with boundary conditions

$$
\bar{A}(T)=1, \bar{B}(T)=0, \bar{C}(T)=0 .
$$

Then, the partial derivatives of $W(t, x, s)$ and $g(t, x, s)$ are given by

$$
\left\{\begin{array}{l}
W_{t}=A^{\prime}(t) x+B^{\prime}(t) s^{-2 \beta}+C^{\prime}(t), W_{x}=A(t), W_{x x}=0, \\
W_{s}=-2 \beta B(t) s^{-2 \beta-1}, W_{s s}=2 \beta(2 \beta+1) B(t) s^{-2 \beta-2}, W_{s x}=0, \\
g_{t}=\bar{A}^{\prime}(t) x+\bar{B}^{\prime}(t) s^{-2 \beta}+\bar{C}^{\prime}(t), g_{x}=\bar{A}(t), g_{x x}=0, \\
g_{s}=-2 \beta \bar{B}(t) s^{-2 \beta-1}, g_{s s}=2 \beta(2 \beta+1) \bar{B}(t) s^{-2 \beta-2}, g_{s x}=0 .
\end{array}\right.
$$

Substituting equations (39)-(42) into equation (32), we obtain 


$$
\begin{aligned}
& {\left[A^{\prime}(t)+\widetilde{r} A(t)\right] x+\left[B^{\prime}(t)-2 \mu_{2} \beta B(t)-2 \sigma_{2}^{2} \gamma \beta^{2} \bar{B}^{2}\right] s^{-2 \beta}+C^{\prime}(t)} \\
& \quad+\sigma_{2}^{2} \beta(2 \beta+1) B(t)+\sup _{\pi \in \Pi} \inf _{Q \in Q}\left\{\frac{\theta_{2}^{2}(t)}{2 \alpha_{2}}+\theta_{2}(t)\left[\pi_{1}(t) \sigma_{2} s^{\beta} A(t)-2 \sigma_{2} \beta B(t) s^{-\beta}\right]\right. \\
& +\frac{\theta_{1}^{2}(t)}{2 \alpha_{1}}+\pi_{2} \theta_{1}(t) \sigma_{1} A(t)-\frac{1}{2} \pi_{1}^{2}(t) \gamma \sigma_{2}^{2} s^{2 \beta} \bar{A}^{2}(t)+\pi_{1}(t)\left[\left(\mu_{2}-r_{1}\right) A(t)+2 \gamma \sigma_{2}^{2} \beta \bar{A}(t) \bar{B}(t)\right] \\
& \left.\quad-\frac{1}{2} \pi_{2}^{2}(t) \gamma \sigma_{1}^{2} \bar{A}^{2}(t)+\pi_{2}(t) \widetilde{r}_{2} A(t)\right\}=0 .
\end{aligned}
$$

According to the first-order optimality condition, $\theta_{1}(t)$ and $\theta_{2}(t)$, solving the minimization problem in equation (43), we obtain

$$
\begin{aligned}
& \theta_{1}^{*}(t)=-\sigma_{1} \alpha_{1} \pi_{2}(t) A(t), \\
& \theta_{2}^{*}(t)=-\sigma_{2} \alpha_{2} \pi_{1}(t) s^{\beta} A(t)+2 \alpha_{2} \beta \sigma_{2} s^{-\beta} B(t) .
\end{aligned}
$$

Plugging (44) and (45) into equation (43) yields

$$
\begin{aligned}
& {\left[A^{\prime}(t)+\widetilde{r} A(t)\right] x+\left[B^{\prime}(t)-2 \mu_{2} \beta B(t)-2 \sigma_{2}^{2} \gamma \beta^{2} \bar{B}^{2}-2 \alpha_{2} \beta^{2} \sigma_{2}^{2} B^{2}(t)\right] s^{-2 \beta}+C^{\prime}(t)+\sigma_{2}^{2} \beta(2 \beta+1) B(t)+\sup _{\pi \in \Pi}} \\
& \left\{-\frac{1}{2} \pi_{1}^{2}\left[\alpha_{2} A^{2}(t)+\gamma \bar{A}^{2}(t)\right] \sigma_{2}^{2} s^{2 \beta}+\pi_{1}(t)\left[\left(\mu_{2}-r_{1}\right) A(t)+2 \gamma \sigma_{2}^{2} \beta \bar{A}(t) \bar{B}(t)+2 \alpha_{2} \sigma_{2}^{2} \beta A(t) B(t)\right]\right. \\
& \left.\quad-\frac{1}{2} \pi_{2}^{2}\left[\alpha_{1} A^{2}(t)+\gamma \bar{A}^{2}(t)\right] \sigma_{1}^{2}+\pi_{2}(t) \widetilde{r}_{2} A(t)\right\}=0 .
\end{aligned}
$$

According to the first-order optimality condition, $\pi_{1}^{*}(t)$ and $\pi_{2}^{*}(t)$, solving the maximization problem in equation (46), we obtain

$$
\pi_{1}^{*}(t)=\frac{\left(\mu_{2}-r_{1}\right) A(t)+2 \gamma \beta \sigma_{2}^{2} \bar{A}(t) \bar{B}(t)+2 \alpha_{2} \sigma_{2}^{2} \beta A(t) B(t)}{\left[\alpha_{2} A^{2}(t)+\gamma \bar{A}^{2}(t)\right] \sigma_{2}^{2}(t) s^{2 \beta}},
$$

$$
\pi_{2}^{*}(t)=\frac{\tilde{r}_{2} A(t)}{\left[\alpha_{1} A^{2}(t)+\gamma \bar{A}^{2}(t)\right] \sigma_{1}^{2}(t)}
$$

Inserting (47) and (48) into equation (46) and after simplifying give

$$
\begin{aligned}
& {\left[A^{\prime}(t)+\widetilde{r} A(t)\right] x+\left\{B^{\prime}(t)-2 \mu_{2} \beta B(t)-2 \sigma_{2}^{2} \gamma \beta^{2} \bar{B}^{2}-2 \alpha_{2} \beta^{2} \sigma_{2}^{2} B^{2}(t)+\frac{\left[\left(\mu_{2}-r_{1}\right) A(t)+2 \gamma \beta \sigma_{2}^{2} \bar{A}(t) \bar{B}(t)+2 \alpha_{2} \sigma_{2}^{2} \beta A(t) B(t)\right]^{2}}{2\left[\alpha_{2} A^{2}(t)+\gamma \bar{A}^{2}(t)\right] \sigma_{2}^{2}}\right\} s^{-2 \beta}} \\
& \quad+\left\{C^{\prime}(t)+\sigma_{2}^{2} \beta(2 \beta+1) B(t)+\frac{\widetilde{r}_{2}^{2} A^{2}(t)}{2\left[\alpha_{1} A^{2}(t)+\gamma \bar{A}^{2}(t)\right] \sigma_{1}^{2}}\right\}=0 .
\end{aligned}
$$


By substituting (40), (42), (44), (45), (47), and (48) into equation (29) yields

$$
\begin{aligned}
& {\left[\bar{A}^{\prime}(t)+\widetilde{r} \bar{A}(t)\right] x+\left\{\bar{B}^{\prime}(t)-2 \mu_{2} \beta \bar{B}(t)-4 \alpha_{2} \beta^{2} \sigma_{2}^{2} B(t) \bar{B}(t)\right.} \\
& \quad+\frac{1}{\left[\alpha_{2} A^{2}(t)+\gamma \bar{A}^{2}(t)\right] \sigma_{2}^{2}}\left[\left[\left(\mu_{2}-r_{1}\right) \bar{A}(t)+2 \sigma_{2}^{2} \alpha_{2} \beta(A(t) \bar{B}(t)+\bar{A}(t) B(t))\right]\right. \\
& \left.\quad \times\left[\left(\mu_{2}-r_{1}\right) A(t)+2 \gamma \beta \sigma_{2}^{2} \bar{A}(t) \bar{B}(t)+2 \alpha_{2} \sigma_{2}^{2} \beta A(t) B(t)\right]\right] \\
& \left.\quad-\frac{\alpha_{2} A(t) \bar{A}(t)\left[\left(\mu_{2}-r_{1}\right) A(t)+2 \gamma \beta \sigma_{2}^{2} \bar{A}(t) \bar{B}(t)+2 \alpha_{2} \sigma_{2}^{2} \beta A(t) B(t)\right]^{2}}{\left[\alpha_{2} A^{2}(t)+\gamma \bar{A}^{2}(t)\right]^{2} \sigma_{2}^{2}}\right\} s^{-2 \beta} \\
& \quad+\left\{\bar{C}^{\prime}(t)+\sigma_{2}^{2} \beta(2 \beta+1) \bar{B}(t)+\frac{\alpha_{1} \widetilde{r}_{2}^{2} A^{3}(t) \bar{A}(t)}{\left[\alpha_{1} A^{2}(t)+\gamma \bar{A}^{2}(t)\right] \sigma_{1}^{2}}-\frac{\left.\alpha_{1} A^{2}(t)+\gamma \bar{A}^{2}(t)\right]^{2} \sigma_{1}^{2}}{\left[\alpha^{2}\right.}\right\}=0 .
\end{aligned}
$$

Thus, we must solve the following ODEs:

$$
\begin{aligned}
& A^{\prime}(t)+\widetilde{r} A(t)=0, A(T)=1, \\
& B^{\prime}(t)-2 \mu_{2} \beta B(t)-2 \sigma_{2}^{2} \gamma \beta^{2} \bar{B}^{2}-2 \alpha_{2} \beta^{2} \sigma_{2}^{2} B^{2}(t) \\
& +\frac{\left[\left(\mu_{2}-r_{1}\right) A(t)+2 \gamma \beta \sigma_{2}^{2} \bar{A}(t) \bar{B}(t)+2 \alpha_{2} \sigma_{2}^{2} \beta A(t) B(t)\right]^{2}}{2\left[\alpha_{2} A^{2}(t)+\gamma \bar{A}^{2}(t)\right] \sigma_{2}^{2}}=0, B(T)=0, \\
& C^{\prime}(t)+\sigma_{2}^{2} \beta(2 \beta+1) B(t)+\frac{\tilde{r}_{2}^{2} A^{2}(t)}{2\left[\alpha_{1} A^{2}(t)+\gamma \bar{A}^{2}(t)\right] \sigma_{1}^{2}}=0, C(T)=0, \\
& \bar{A}^{\prime}(t)+\widetilde{r} \bar{A}(t)=0, \bar{A}(T)=1, \\
& \bar{B}^{\prime}(t)-2 \mu_{2} \beta \bar{B}(t)-4 \alpha_{2} \beta^{2} \sigma_{2}^{2} B(t) \bar{B}(t) \\
& +\frac{1}{\left[\alpha_{2} A^{2}(t)+\gamma \bar{A}^{2}(t)\right] \sigma_{2}^{2}}\left[\left[\left(\mu_{2}-r_{1}\right) \bar{A}(t)+2 \sigma_{2}^{2} \alpha_{2} \beta(A(t) \bar{B}(t)+\bar{A}(t) B(t))\right]\right. \\
& \left.\times\left[\left(\mu_{2}-r_{1}\right) A(t)+2 \gamma \beta \sigma_{2}^{2} \bar{A}(t) \bar{B}(t)+2 \alpha_{2} \sigma_{2}^{2} \beta A(t) B(t)\right]\right] \\
& -\frac{\alpha_{2} A(t) \bar{A}(t)\left[\left(\mu_{2}-r_{1}\right) A(t)+2 \gamma \beta \sigma_{2}^{2} \bar{A}(t) \bar{B}(t)+2 \alpha_{2} \sigma_{2}^{2} \beta A(t) B(t)\right]^{2}}{\left[\alpha_{2} A^{2}(t)+\gamma \bar{A}^{2}(t)\right]^{2} \sigma_{2}^{2}}=0, \bar{B}(T)=0 \text {, } \\
& \bar{C}^{\prime}(t)+\sigma_{2}^{2} \beta(2 \beta+1) \bar{B}(t)+\frac{\tilde{r}_{2}^{2} A(t) \bar{A}(t)}{\left[\alpha_{1} A^{2}(t)+\gamma \bar{A}^{2}(t)\right] \sigma_{1}^{2}}-\frac{\alpha_{1} \widetilde{r}_{2}^{2} A^{3}(t) \bar{A}(t)}{\left[\alpha_{1} A^{2}(t)+\gamma \bar{A}^{2}(t)\right]^{2} \sigma_{1}^{2}}=0, \bar{C}(T)=0 .
\end{aligned}
$$
obtain

First, solving equations (51) and (54), respectively, we

$$
\begin{aligned}
& A(t)=e^{\tilde{r}(T-t)}, \\
& \bar{A}(t)=e^{\widetilde{r}(T-t)} .
\end{aligned}
$$

By substituting (57) and (58) into (52) and (55), we can obtain $B(t)$ and $\bar{B}(t)$ which are the solutions of ODEs (38).

Inserting (57), (58), and $\bar{B}(t)$ into equation (56), we obtain the solution to equation (56) which is given by 


$$
\bar{C}(t)=\frac{\gamma \widetilde{r}_{2}^{2}(T-t)}{\left(\alpha_{1}+\gamma\right)^{2} \sigma_{1}^{2}}+\sigma_{2}^{2} \beta(2 \beta+1) \int_{t}^{T} \bar{B}(s) \mathrm{d} s .
$$

By substituting (57), (58), and $B(t)$ into equation (53), and solving it, we obtain

$$
C(t)=\frac{\widetilde{r}_{2}^{2}}{2\left(\alpha_{1}+\gamma\right) \sigma_{1}^{2}}(T-t)+\sigma_{2}^{2} \beta(2 \beta+1) \int_{t}^{T} B(s) \mathrm{d} s .
$$

Inserting (57), (58), $B(t)$, and $\bar{B}(t)$ into (47), we can obtain (33). By substituting (57) and (58) into (48), we can obtain (34). Plugging (33) into (45) and (34) into (44), we yield (36) and (35), respectively.

Based on Theorem 2 and the notation setting (8), we have the following theorem.

Theorem 3. For the wealth process (7), the optimal robust time-consistent investment strategies to robust $M V$ problem (19) are given by

$$
\begin{gathered}
u_{1}^{*}(t)=\frac{\left(\mu_{2}-r_{1}\right)+2 \gamma \beta \sigma_{2}^{2} \bar{B}(t)+2 \alpha_{2} \beta \sigma_{2}^{2} B(t)}{x\left(\alpha_{2}+\gamma\right) \sigma_{2}^{2} s^{2 \beta}} e^{-\widetilde{r}(T-t),} \\
u_{2}^{*}(t)=\frac{\widetilde{r}_{2}}{x\left(\alpha_{1}+\gamma\right) \sigma_{1}^{2}} e^{-\widetilde{r}(T-t)}+1 .
\end{gathered}
$$

Remark 2. From the expressions of optimal robust timeconsistent investment strategies (61) and (62), we note that $u_{1}^{*}(t)$ is related to the appreciation rate $\mu_{2}$ and price volatility $\sigma_{2}$ of the risky asset and the current wealth $x$, whereas $u_{2}^{*}(t)$ is related to the parameters $\mu_{1}$ and $\sigma_{1}$ of the inflation level and the current wealth $x$. This difference between $u_{1}^{*}(t)$ and $u_{2}^{*}(t)$ is mainly because the inflation price process and the risky asset price process are independent in our model assumption.

\section{Numerical Experiments}

This section is devoted to providing the influences of model parameters on the optimal time-consistent investment strategy and utility loss function by numerical experiments. In the following, unless otherwise stated, we choose the basic parameter setting as given in Table 1 .

5.1. The Influences of the Parameters on $u_{1}^{*}(t)$. We first present the effects of model parameters on $u_{1}^{*}(t)$, where $u_{1}^{*}(t)$ is determined by $(61)$.

Let $s \in[2,10]$; Figure 1 shows the effect of $s$ on $u_{1}^{*}(t)$. From Figure 1, it is evident that $u_{1}^{*}(t)$ is a decreasing function of $s$, which describes the price of the risky asset. Form the meaning of $s$, we know that larger $s$ implies that the investment risk becomes greater. Therefore, as $s$ increases, the AAI will wish to invest less in the risky asset.

Figure 2 presents two curves about the effect of $\beta$ on $u_{1}^{*}(t)$, where parameter $\beta \in[0.1,0.6]$. From Figure 2 , we can see that when $s=2, u_{1}^{*}(t)$ is a decreasing function in $\beta$, while
TABLE 1: Values of model parameters.

\begin{tabular}{cccccccccccccc}
\hline$\mu_{1}$ & $\sigma_{1}$ & $r_{1}$ & $\mu_{2}$ & $\sigma_{2}$ & $r_{2}$ & $\gamma$ & $x$ & $T$ & $t$ & $\beta$ & $\mathrm{s}$ & $\alpha_{1}$ & $\alpha_{2}$ \\
\hline 0.05 & 0.3 & 0.03 & 0.08 & 0.25 & 0.02 & 2 & 10 & 10 & 9 & 0.1 & 2 & 2 & 1 \\
\hline
\end{tabular}

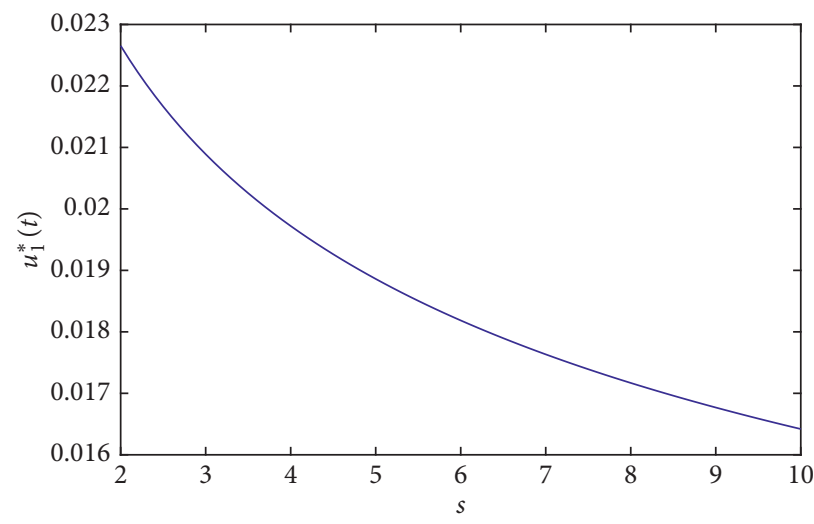

Figure 1: Effect of $s$ on $u_{1}^{*}(t)$.

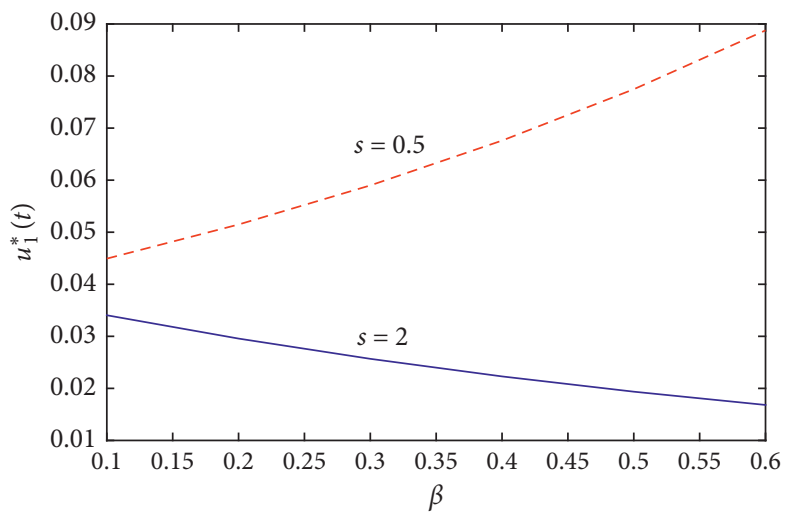

Figure 2: Effect of $\beta$ on $u_{1}^{*}(t)$.

if $s=0.5, u_{1}^{*}(t)$ is an increasing function in $\beta$. When $s=2$ $(s=0.5)$, the larger $\beta$ is, the greater (less) $s^{\beta}$ will be and hence the greater (less) the risk will be; thus, the AAI will wish to invest less (greater) in the risky asset.

Let $\mu_{2} \in[0.05,0.1]$; we present the effect of $\mu_{2}$ on $u_{1}^{*}(t)$ in Figure 3. From Figure 3, it is clear that $u_{1}^{*}(t)$ is increasing with respect to $\mu_{2}$, which is the appreciation rate of the risky asset. Larger $\mu_{2}$ implies that the expected income of the risky asset becomes greater. As a result, the AAI will wish to invest more in the risky asset.

Let $\sigma_{2} \in[0.2,0.6]$; Figure 4 displays the effect of $\sigma_{2}$ on $u_{1}^{*}(t)$. From Figure 4 , we can observe that $u_{1}^{*}(t)$ is a decreasing function of $\sigma_{2}$. Recall that $\sigma_{2}$ is the price volatility; therefore, with the increase of $\sigma_{2}$, the investment risk will increase. Thus, the AAI will wish to invest less in the risky asset.

Figure 5 illustrates the effect of the risk-free interest rate $r_{1}$ on $u_{1}^{*}(t)$, where $r_{1} \in[0.02,0.06]$. This figure shows that $u_{1}^{*}(t)$ is a decreasing function with respect to $r_{1}$. Larger $r_{1}$ implies that the expected income of the risk-free asset 


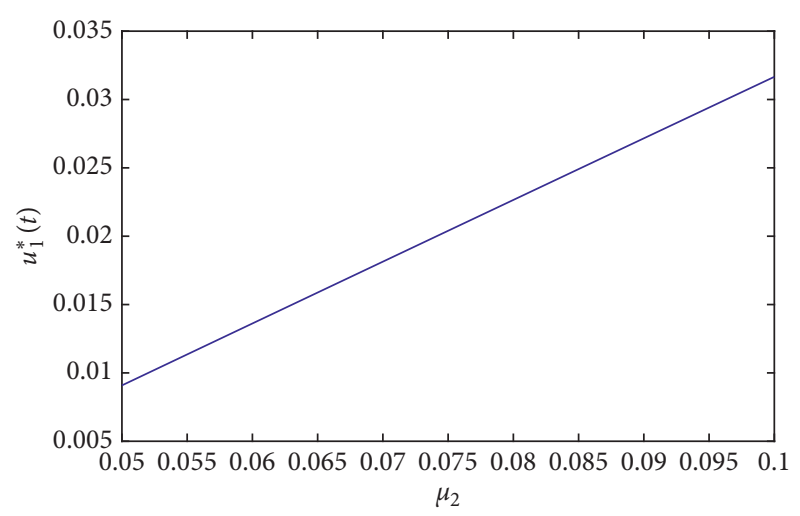

Figure 3: Effect of $\mu_{2}$ on $u_{1}^{*}(t)$.

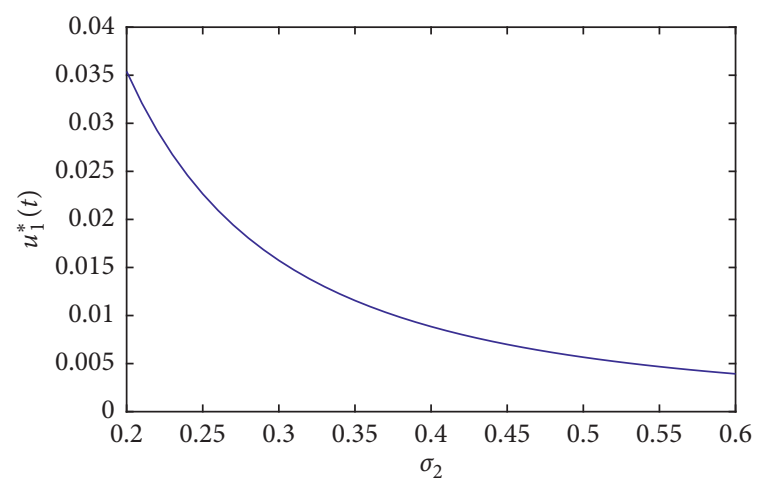

Figure 4: Effect of $\sigma_{2}$ on $u_{1}^{*}(t)$.

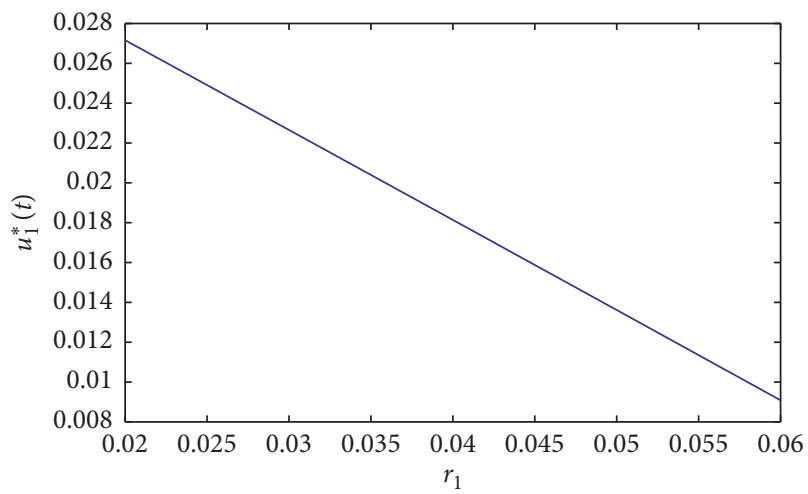

FiguRE 5: Effect of $r_{1}$ on $u_{1}^{*}(t)$.

becomes greater. A natural result is that the AAI will invest more in the risk-free asset. That is to say, the AAI will invest less in the risky asset.

Figure 6 discloses that $u_{1}^{*}(t)$ is a decreasing function of $\alpha_{2}$, where $\alpha_{2} \in[1,6]$. Recall that $\alpha_{2}$ stands for the AAI's ambiguity aversion. Larger $\alpha_{2}$ implies that the model uncertainty becomes greater. Hence, the AAI will invest less in the risky asset.

Figure 7 shows the effect of $\gamma$ on $u_{1}^{*}(t)$, where $\gamma \in[2,8]$. From Figure 7, we see that $u_{1}^{*}(t)$ is a decreasing function with respect to $\gamma$. This is obvious. Since $\gamma$ is the risk-aversion parameter, larger $\gamma$ implies that the less aggressive the AAI will be. Therefore, the AAI will invest less in the risky asset.

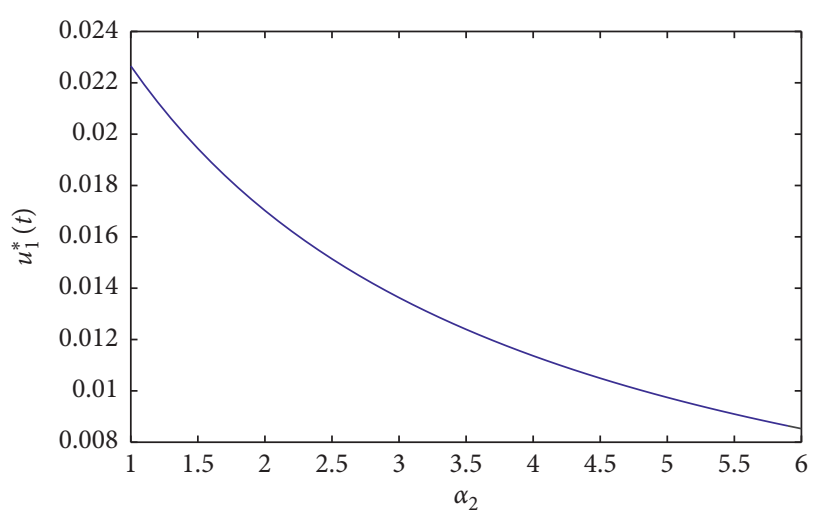

Figure 6: Effect of $\alpha_{2}$ on $u_{1}^{*}(t)$.

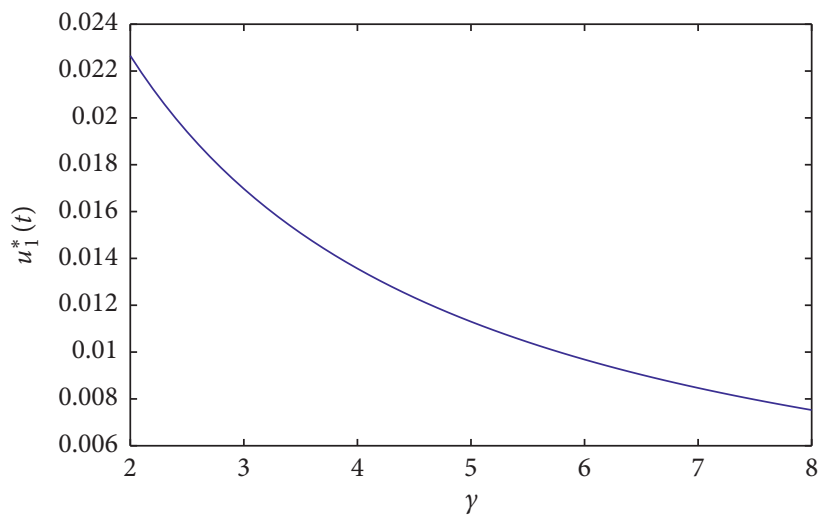

FIgURE 7: Effect of $\gamma$ on $u_{1}^{*}(t)$.

Figure 8 reveals that $u_{1}^{*}(t)$ is increasing with respect to $t$, where $t \in[2,9]$. This implies that, as the termination time of the investment approaches, the AAI will invest more in the risky assets.

5.2. The Influences of the Parameters on $u_{2}^{*}(t)$. Now, we illustrate the effects of model parameters on the optimal robust time-consistent investment strategy $u_{2}^{*}(t)$, where $u_{2}^{*}(t)$ is determined by $(62)$.

Differentiating (62) with respect to $\mu_{1}$, we obtain

$$
\frac{\partial u_{2}^{*}(t)}{\partial \mu_{1}}=\frac{1}{x\left(\alpha_{1}+\gamma\right) \sigma_{1}^{2}} e^{-\tilde{r}(T-t)}>0,
$$

that is, $u_{2}^{*}(t)$ is increasing with respect to $\mu_{1}$. This implies that, as the interest rate of the inflation-indexed bond increases, the AAI should retain more investments in the inflation-indexed bond.

Differentiating (62) with respect to $\sigma_{1}$, we have

$$
\frac{\partial u_{2}^{*}(t)}{\partial \sigma_{1}}=-\frac{2\left(r_{2}+\mu_{1}-r_{1}\right)}{x\left(\alpha_{1}+\gamma\right) \sigma_{1}^{3}} e^{-\tilde{r}(T-t)}<0,
$$

so we know that $u_{2}^{*}(t)$ is decreasing with respect to $\sigma_{1}$. Recall that $\sigma_{1}$ is the volatility of the inflation-indexed bond. Therefore, larger $\sigma_{1}$ implies that the investment risk in the inflation-indexed bond becomes larger. Hence, the AAI retains less investments in the inflation-indexed bond. 


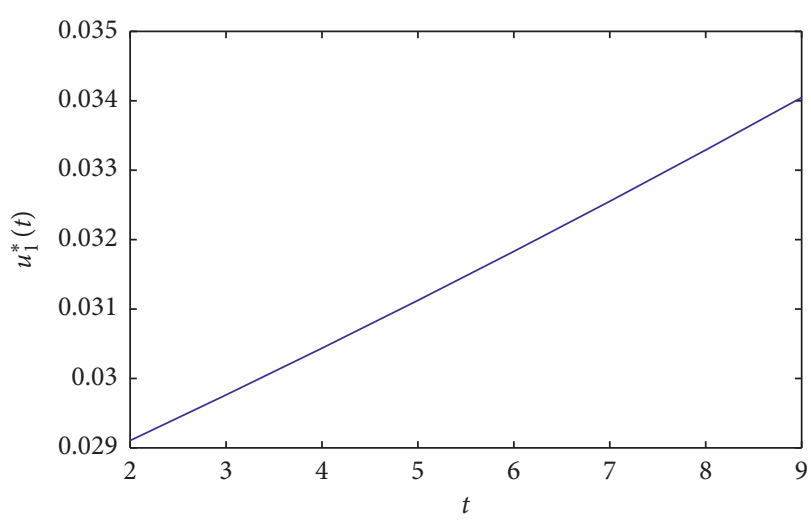

Figure 8: Effect of $t$ on $u_{1}^{*}(t)$.

Differentiating (62) with respect to $r_{1}$, we obtain

$$
\frac{\partial u_{2}^{*}(t)}{\partial r_{1}}=-\frac{1}{x\left(\alpha_{1}+\gamma\right) \sigma_{1}^{2}} e^{-\tilde{r}(T-t)}<0,
$$

which implies that $u_{2}^{*}(t)$ is decreasing with respect to $r_{1}$. The reason is similar to what we have explained in Figure 5.

In the following, we analyze the effect of $r_{2}$ on $u_{2}^{*}(t)$ by the numerical experiment. We set $\mu_{1}=0.02, \sigma_{1}=0.04, \alpha_{1}=$ 10, $\gamma=2, x=10, T=10, t=5, r_{2} \in[0.01,0.03]$, and the result is shown in Figure 9. From Figure 9, we can observe that $u_{2}^{*}(t)$ is an increasing function with respect to $r_{2}$. Recall that $r_{2}$ stands for the real interest rate of the inflationindexed bond. Hence, the larger $r_{2}$ is, the more the AAI will invest in the inflation-indexed bond.

5.3. The Influences of Model Parameters on Utility Loss Function. In Sections 5.1 and 5.2, we studied the case under the robust model. This part uses numerical experiments to investigate the influences of model parameters on utility loss function.

First, we give some results under the ambiguity-neutral case.

For an ambiguity-neutral optimization problem, all admissible investment strategies $\bar{\pi}(t)$ are denoted by $\bar{\Pi}$. Then, the wealth process becomes

$$
\begin{aligned}
\mathrm{d} X_{t}^{\bar{\pi}}= & {\left[\widetilde{r} X_{t}^{\bar{\pi}}+\bar{\pi}_{1}(t)\left(\mu_{2}-r_{1}\right)+\bar{\pi}_{2}(t) \widetilde{r}_{2}\right] \mathrm{d} t } \\
& +\bar{\pi}_{1}(t) \sigma_{2} S^{\beta}(t) \mathrm{d} W_{2}(t)+\bar{\pi}_{2}(t) \sigma_{1} \mathrm{~d} W_{1}(t),
\end{aligned}
$$

and the objective function is as follows:

$$
\sup _{\bar{\pi} \in \bar{\Pi}}\left\{E_{t, x, s}\left[X_{T}^{\bar{\pi}}\right]-\frac{\gamma}{2} \operatorname{Var}_{t, x, s}\left[X_{T}^{\bar{\pi}}\right]\right\} .
$$

Similar to what we have proved in Theorem 2, we can obtain the following theorem.

Theorem 4. For problem (67), the optimal time-consistent investment strategies are given by

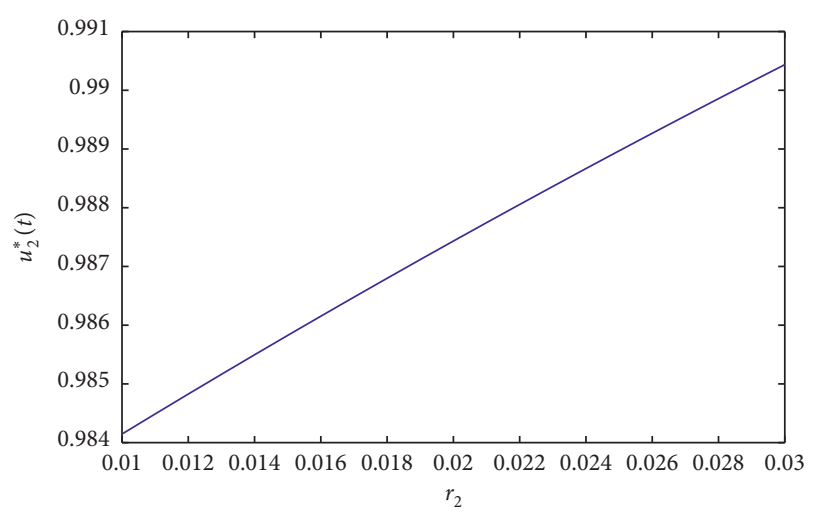

Figure 9: Effect of $r_{2}$ on $u_{2}^{*}(t)$.

$$
\begin{aligned}
& \bar{\pi}_{1}^{*}(t)=\frac{\left(\mu_{2}-r_{1}\right)+2 \gamma \beta \sigma_{2}^{2} B_{2}(t)}{\gamma \sigma_{2}^{2} s^{2 \beta}} e^{-\widetilde{r}(T-t)}, \\
& \bar{\pi}_{2}^{*}(t)=\frac{\widetilde{r}_{2}}{\gamma \sigma_{1}^{2}} e^{-\widetilde{r}(T-t)} .
\end{aligned}
$$

Furthermore, the optimal value function is given by

$$
W_{1}(t, x, s)=x e^{\tilde{r}(T-t)}+B_{1}(t) s^{-2 \beta}+C_{1}(t),
$$

where

$$
\begin{aligned}
& B_{1}(t)=e^{-2 \mu_{2} \beta(T-t)} \int_{t}^{T} f_{1}(s) e^{2 \mu_{2} \beta(T-s)} \mathrm{d} s, \\
& B_{2}(t)=\frac{\left(\mu_{2}-r_{1}\right)^{2}}{2 r_{1} \beta \gamma \sigma_{2}^{2}}\left[1-e^{-2 r_{1} \beta(T-t)}\right] \\
& f_{1}(t)=2 \beta\left(\mu_{2}-r_{1}\right) B_{2}(t)+\frac{\left(\mu_{2}-r_{1}\right)^{2}}{2 \gamma \sigma_{2}^{2}}, \\
& C_{1}(t)=\frac{\widetilde{r}_{2}^{2}}{2 \gamma \sigma_{1}^{2}}(T-t)+\sigma_{2}^{2} \beta(2 \beta+1) \int_{t}^{T} B_{1}(s) \mathrm{d} s .
\end{aligned}
$$

Based on Theorems 2 and 4, we can define the utility loss function as

$$
L(t)=1-\frac{W_{1}(t, x, s)}{W(t, x, s)}
$$

In the following, we investigate the effects of model parameters on $L(t)$.

(i) The influence of ambiguity aversion on the utility loss: Let $\alpha_{2}=4$ and $\alpha_{1}=2,3,4,5,6,7,8$; we can obtain $W_{1}(t, x, s)=10.2370$. The values of $W(t, x, s)$ and $L(t)$ are presented in Table 2. From Table 2, we know that the utility loss function is a decreasing function in $\alpha_{1}$, where $\alpha_{1}$ stands for the ambiguity aversion which arises from the inflation-indexed bond. By comparing the AAI's utility loss at different $\alpha_{1}$, we find that the more ambiguity averse the AAI is, the less conservative investment strategy the AAI will be (because $W(t, x, s)$ is decreasing in $\left.\alpha_{1}\right)$. Because AAI's 
TABLe 2: Effect of $\alpha_{1}$ on $W(t, x, s)$ and $L(t)$.

\begin{tabular}{lcccccrr}
\hline$\alpha_{1}$ & 2 & 3 & 4 & 5 & 6 & 7 & 8 \\
\hline$W(t, x, s)$ & 11.7217 & 11.7212 & 11.7209 & 11.7207 & 11.7205 & 11.7204 & 11.7202 \\
$L(t)$ & 0.12666 & 0.12663 & 0.1266 & 0.12658 & 0.12657 & 0.12656 & 0.12655 \\
\hline
\end{tabular}

TABle 3: Effect of $\alpha_{2}$ on $W(t, x, s)$ and $L(t)$.

\begin{tabular}{lccccccc}
\hline$\alpha_{2}$ & 2 & 3 & 4 & 5 & 6 & 7 & 8 \\
\hline$W(t, x, s)$ & 11.0307 & 11.0386 & 11.0429 & 11.0455 & 11.0472 & 11.0484 & 11.0492 \\
$L(t)$ & 0.0719 & 0.0726 & 0.0730 & 0.0732 & 0.0733 & 0.0734 & 0.0735 \\
\hline
\end{tabular}

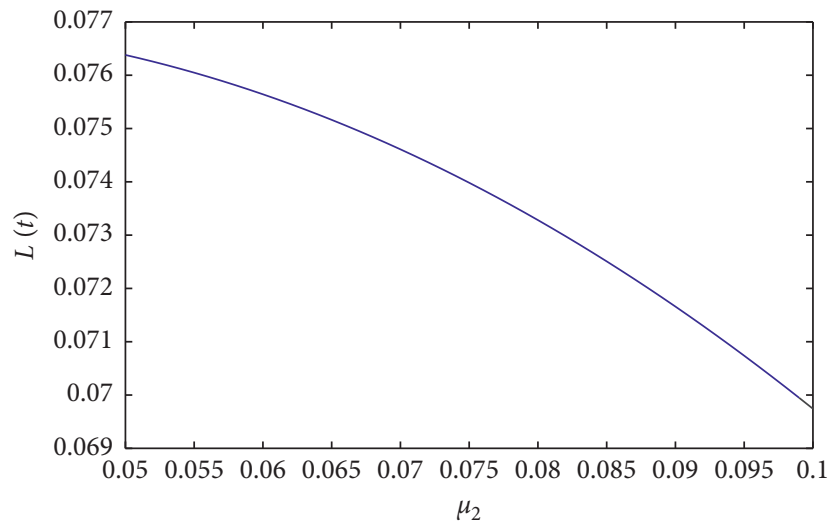

Figure 10: Effect of $\mu_{2}$ on $L(t)$.

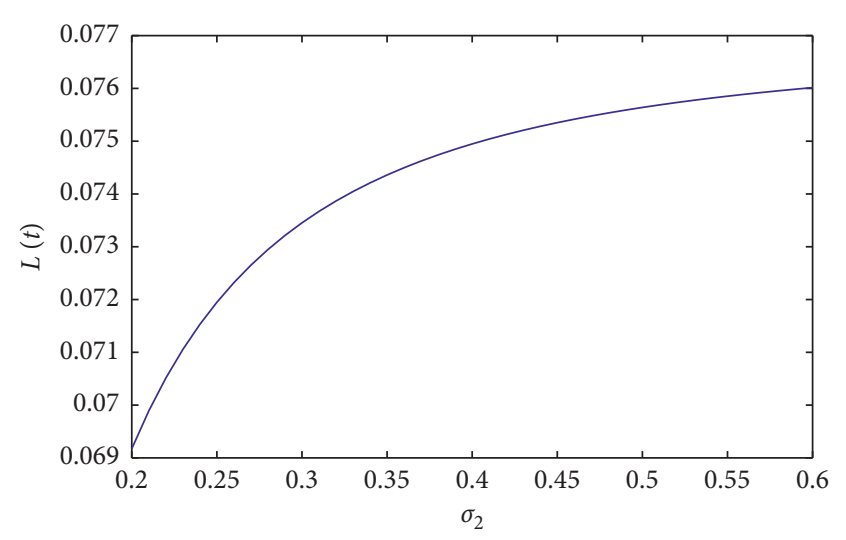

Figure 11: Effect of $\sigma_{2}$ on $L(t)$.

investment is robust, he will invest more money in the risk-free asset, so AAI will suffer less utility loss.

Let $\alpha_{1}=4$ and $\alpha_{2}=2,3,4,5,6,7,8$; we have $W_{1}(t, x, s)=10.2370$. The values of $W(t, x, s)$ and $L(t)$ are presented in Table 3 . From Table 3 , it is evident that the utility loss function is an increasing function in $\alpha_{2}$, where $\alpha_{2}$ stands for the ambiguity aversion which arises from the risky asset.

(ii) The influence of market parameters on the utility loss:

Figure 10 discloses that $L(t)$ is a decreasing function with respect to $\mu_{2}$, where $\mu_{2} \in[0.05,0.1]$. This indicates that the utility loss increases as the appreciation rate of the risky asset diminishes.

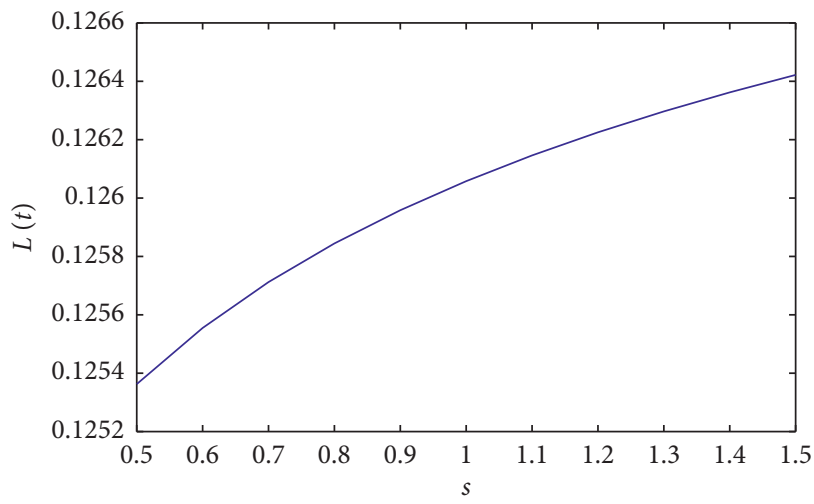

Figure 12: Effect of $s$ on $L(t)$.

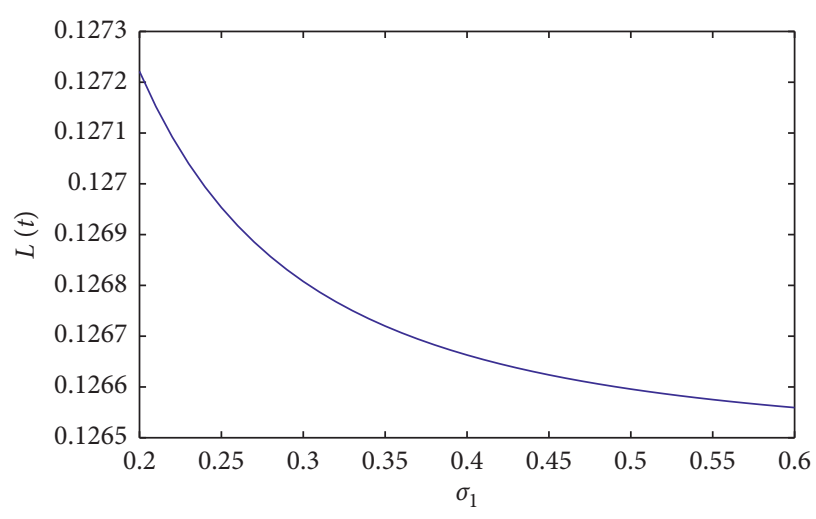

Figure 13: Effect of $\sigma_{1}$ on $L(t)$.

Figure 11 reveals that $L(t)$ is an increasing function with regard to $\sigma_{2}$, where $\sigma_{2} \in[0.2,0.6]$. Larger $\sigma_{2}$ implies that the investment risk becomes greater, and thus, the AAI will suffer from more utility losses.

Let $s \in[0.5,1.5]$; Figure 12 gives the influence of parameter s on the utility loss. From Figure 12, we can observe that $L(t)$ is an increasing function of $s$. The larger the $s$ is, the larger the price volatility will be, that is, the greater the risk will be, and hence, the AAI will suffer from more utility losses.

Let $\sigma_{1} \in[0.2,0.6]$; Figure 13 shows that the utility loss is a decreasing function of $\sigma_{1}$. Comparing Figures 11 with 13, it is clear that the effect of the volatilities of 
the risky asset and the inflation on the utility loss is opposite.

\section{Conclusions}

We have investigated a robust time-consistent MV strategy selection under the inflation risk for an AAI. The AAI doubts the model setting under the original probability measure. Then, he seeks a set of alternative probability measures, which are absolutely continuous to the original probability measure. Furthermore, we modify the model setting under the alternative probability measures. We form the AAI's objective under the framework of the robust MV criterion. As we all know, this problem is time-inconsistent. To solve this time-inconsistent problem, we seek the equilibrium strategy from the game framework. The equilibrium strategy is time-consistent. By using the stochastic optimal control technique, we derive the closed-form solutions for the optimal time-consistent investment strategy and the optimal value function. Finally, the influences of model parameters on the optimal time-consistent investment strategy and utility loss function are examined through numerical experiments.

Compared with Zeng et al. [12], Zheng et al. [28], Maenhout $[34,35]$, and other studies about the robust MV problem, the inflation risk and the CEV model are simultaneously introduced in this article. In addition, the influence of the model parameters of the inflation on the optimal time-consistent investment strategy and the utility loss are systematic analysis by numerical experiments.

It may be interesting to extend the current framework to that with dividend and state-dependent risk-aversion function or that in a partial information market in the future research. For these future research topics, we may need to adopt the methods used in Bjök et al. [14], Yu et al. [37], and Raychaudhury and Pal [38].

\section{Data Availability}

The data used to support the findings of this study are available upon request to the author.

\section{Conflicts of Interest}

The author declares that there are no conflicts of interest regarding the publication of this paper.

\section{References}

[1] H. Markowitz, "Portfolio selection," The Journal of Finance, vol. 7, no. 1, pp. 77-91, 1952.

[2] D. Li and W.-L. Ng, "Optimal dynamic portfolio selection: multiperiod mean-variance formulation," Mathematical Finance, vol. 10, no. 3, pp. 387-406, 2000.

[3] X. Y. Zhou and D. Li, "Continuous-time mean-variance portfolio selection: a stochastic LQ framework," Applied Mathematics and Optimization, vol. 42, no. 1, pp. 19-33, 2000.

[4] M. Dai, Z. Q. Xu, and X. Y. Zhou, "Continuous-time markowitz's model with transaction costs," SIAM Journal on Financial Mathematics, vol. 1, no. 1, pp. 96-125, 2010.
[5] Z. Sun, K. C. Yuen, and J. Guo, “A BSDE approach to a class of dependent risk model of mean-variance insurers with stochastic volatility and no-short selling," Journal of Computational and Applied Mathematics, vol. 366, Article ID 112413, 2020.

[6] Z. Sun, X. Zhang, and K. C. Yuen, "Mean-variance asset-liability management with affine diffusion factor process and a reinsurance option," Scandinavian Actuarial Journal, vol. 2020, no. 3, pp. 218-244, 2020.

[7] R. H. Strotz, "Myopia and inconsistency in dynamic utility maximization," The Review of Economic Studies, vol. 23, no. 3, pp. 165-180, 1955.

[8] I. Ekeland, O. Mbodji, and T. A. Pirvu, "Time-consistent portfolio management," SIAM Journal on Financial Mathematics, vol. 3, no. 1, pp. 1-32, 2012.

[9] T. Björk and A. Murgoci, "A general theory of Markovian time inconsistent stochastic control problems," in Working Paper, Stockholm School of Economics, Stockholm, Sweden, 2010.

[10] Z. Li, Y. Zeng, and Y. Lai, "Optimal time-consistent investment and reinsurance strategies for insurers under Heston's SV model," Insurance: Mathematics and Economics, vol. 51, no. 1, pp. 191-203, 2012.

[11] P. Yang, "Time-consistent mean-variance reinsurance-investment in a jump-diffusion financial market," Optimization, vol. 66, no. 5, pp. 737-758, 2017.

[12] Y. Zeng, D. Li, and A. Gu, "Robust equilibrium reinsuranceinvestment strategy for a mean-variance insurer in a model with jumps," Insurance: Mathematics and Economics, vol. 66, pp. 138-152, 2016.

[13] P. Yang, Z. Chen, and Y. Xu, "Time-consistent equilibrium reinsurance-investment strategy for $\mathrm{n}$ competitive insurers under a new interaction mechanism and a general investment framework," Journal of Computational and Applied Mathematics, vol. 374, Article ID 112769, 2020.

[14] T. Björk, A. Murgoci, and X. Zhou, "Mean variance portfolio optimization with state dependent risk aversion," Math Finance, vol. 24, no. 1, pp. 1-24, 2014.

[15] C. Czichowsky, "Time-consistent mean-variance portfolio selection in discrete and continuous time," Finance and Stochastics, vol. 17, no. 2, pp. 227-271, 2013.

[16] M. T. Kronborg and M. Steffensen, "Inconsistent investment and consumption problems," Applied Mathematics \& Optimization, vol. 71, no. 3, pp. 473-515, 2015.

[17] M. J. Brennan and Y. Xia, "Dynamic asset allocation under inflation," The Journal of Finance, vol. 57, no. 3, pp. 1201-1238, 2002.

[18] N.-W. Han and M.-W. Hung, "Optimal asset allocation for DC pension plans under inflation," Insurance: Mathematics and Economics, vol. 51, no. 1, pp. 172-181, 2012.

[19] M. Kwak and B. H. Lim, "Optimal portfolio selection with life insurance under inflation risk," Journal of Banking \& Finance, vol. 46, pp. 59-71, 2014.

[20] Y. Li, S. Wang, Y. Zeng, and H. Qiao, "Equilibrium investment strategy for a DC plan with partial information and mean-variance criterion," IEEE Systems Journal, vol. 11, no. 3, pp. 1492-1504, 2016.

[21] J. C. Cox and S. A. Ross, "The valuation of options for alternative stochastic processes," Journal of Financial Economics, vol. 3, no. 1-2, pp. 145-166, 1976.

[22] J. Gao, "Optimal investment strategy for annuity contracts under the constant elasticity of variance (CEV) model," Insurance: Mathematics and Economics, vol. 45, no. 1, pp. 9-18, 2009. 
[23] M. Gu, Y. Yang, S. Li, and J. Zhang, "Constant elasticity of variance model for proportional reinsurance and investment strategies," Insurance: Mathematics and Economics, vol. 46, no. 3, pp. 580-587, 2010.

[24] X. Lin and Y. Li, "Optimal reinsurance and investment for a jump diffusion risk process under the CEV model," North American Actuarial Journal, vol. 15, no. 3, pp. 417-431, 2011.

[25] Z. Liang, K. C. Yuen, and K. C. Cheung, "Optimal reinsurance-investment problem in a constant elasticity of variance stock market for jump-diffusion risk model," Applied Stochastic Models in Business and Industry, vol. 28, no. 6, pp. 585-597, 2012.

[26] X. Lin and Y. Qian, "Time-consistent mean-variance reinsurance-investment strategy for insurers under CEV model," Scandinavian Actuarial Journal, vol. 2016, no. 7, pp. 646-671, 2016.

[27] D. Li, R. Chen, and C. Li, "Optimal reinsurance-investmentproblem under a CEV model: stochastic differential game formulation," Mathematical Problems in Engineering, vol. 2020, Article ID 7265121, 19 pages, 2020.

[28] X. Zheng, J. Zhou, and Z. Sun, "Robust optimal portfolio and proportional reinsurance for an insurer under a CEV model," Insurance: Mathematics and Economics, vol. 67, pp. 77-87, 2016.

[29] D. C. Emanuel and J. D. Macbeth, "Further results on the constant elasticity of variance call option pricing model," The Journal of Financial and Quantitative Analysis, vol. 17, no. 4, pp. 533-554, 1982.

[30] W. Yu, Y. Yong, G. Guan, Y. Huang, W. Su, and C. Cui, "Valuing guaranteed minimum death benefits by cosine series expansion," Mathematics, vol. 7, no. 9, p. 835, 2019.

[31] Z. Zhang, Y. Yong, and W. Yu, "Valuing equity-linked death benefits in general exponential Lévy models," Journal of Computational and Applied Mathematics, vol. 365, Article ID 112377, 2020.

[32] G.-E. Espinosa and N. Touzi, "Optimal investment under relative performance concerns," Mathematical Finance, vol. 25, no. 2, pp. 221-257, 2015.

[33] W. Yu, F. Wang, Y. Huang, and H. Liu, "Social optimal mean field control problem for population growth model," Asian Journal of Control, pp. 1-8, 2019.

[34] P. J. Maenhout, "Robust portfolio rules and asset pricing," Review of Financial Studies, vol. 17, no. 4, pp. 951-983, 2004.

[35] P. J. Maenhout, "Robust portfolio rules and detection-error probabilities for a mean-reverting risk premium," Journal of Economic Theory, vol. 128, no. 1, pp. 136-163, 2006.

[36] Z. Chen and P. Yang, "Robust optimal reinsurance-investment strategy with price jumps and correlated claims," Insurance: Mathematics and Economics, vol. 92, pp. 27-46, 2020.

[37] W. Yu, P. Guo, Q. Wang et al., "On a periodic capital injection and barrier dividend strategy in the compound Poisson risk model," Mathematics, vol. 8, no. 4, p. 511, 2020.

[38] C. Raychaudhury and D. Pal, "Usefulness of graph vertex complexity and class partial information content in explaining gas phase thermal entropy of chemical compounds," Journal of Mathematical Chemistry, vol. 58, no. 5, pp. 887-892, 2020. 